\title{
A practical approach to manage data communication security
}

first author and point of contact:

\author{
P.H. Samwel \\ Rabofacet, ZL-R-142 \\ P.O. Box 17100, 3500 HG Utrecht, The Netherlands \\ E-mail: P.H.Samwel@rf.rabobank.nl
}

second author:

\section{M.E.M. Spruit}

Delft University of Technology, Department of Information Systems

P.O.Box 356, 2600 AJ Delft, The Netherlands

E-mail: spruit@is.twi.tudelft.nl

Affiliation: Rabobank

Key words: Data communication, Network security, Security management, Security classification, IT Audit, EDP Audit, Availability, Integrity, Confidentiality

Abstract: This paper describes a practical approach to manage the security of data communication infrastructures. The approach is based upon the classification of network segments and the description of the relation between segments. This will result in a clear view of the security characteristics of all relevant data communication paths, even in large networks. This view is useful for data communication product managers, information system owners and IT auditors. The examples in this paper are based upon an implementation of this approach for the Rabobank IP network infrastructure. The examples however reflect a simplified version of this network for illustration purposes. 


\section{INTRODUCTION}

The network infrastructure is an essential component in the security of computer applications. The entire data communication path between users and the application, as well as the data communication path between application components, is relevant for the integrity and the confidentiality of the information flows and the availability of the application.

Due to the complexity of networks, application owners usually don't have a clear picture of the security characteristics of the data communication paths that are relevant for their application. Furthermore, network infrastructure owners usually don't know the full range of information flows that pass through the sections they are responsible for. Hence they both don't have a clear view of the security measures that should be implemented in this infrastructure, given the nature of the applications using the infrastructure. However, traditional risk analysis (for example CRAMM [CCTA]) requires accurate information on the relation between business processes and information systems on the one hand, and between information systems and the underlying objects on the other hand. As this information generally cannot be made available in a complex network situation, neither application nor infrastructure owners know whether and what additional measures should be implemented in either the application or the infrastructure. A widely used alternative for complex networks is to implement the security measures which are necessary to comply to a certain baseline (for example the standard BS7799, Code of Practice [COP]). This only works however if every relevant section of the network complies to the baseline completely, and if the applications using the infrastructure do not require additional security measures. In practice these conditions generally will not be met. Hence the baseline approach isn't adequate either.

As a result of the above, organisations probably implement security structures with gaps. At the same time they may implement measures that are not necessary or not effective, due to other parts of the infrastructure that are less secure. All in all this results in a security structure which is inadequate and unnecessary expensive.

A solution to the above problem can be found by evaluating the implemented security measures in the infrastructure and subsequently classifying the various parts of the network. By relating the security classification of the relevant parts of the network to the security classification of applications, one can fill the missing link between on the 
one hand security needs of applications and business processes and on the other hand security measures in the infrastructure. This paper describes a practical approach to implement such a solution.

\section{DEFINING THE CLASSIFICATION LEVELS}

To identify whether a certain data communication path in a network infrastructure meets the security requirements for a specific application, one has to figure out which part of the infrastructure is used by the application. If that relation is known, one can assess whether the security measures of the infrastructure cover the relevant security requirements sufficiently. Because different applications use different parts of the infrastructure, the relation between application and parts of infrastructure generally cannot be done on a one to one basis.

A suitable segmentation of the network infrastructure however, can be used as an intermediate to make the above relation manageable. To achieve this, the network infrastructure is divided into segments which on the one hand offer a certain security level with respect to the quality aspects availability, integrity and confidentiality, and on the other hand is within the area of responsibility of only one organisational unit. If a certain minimal security level is applicable all over the given segment, then one can assign a corresponding classification level to the segment. [ECC91] and [NCSC85] describe security classification standards for computer systems. A similar way of classification can be used for classifying segments of a network infrastructure. One can choose for a classification scheme which is specific for the organisation, or a classification scheme which can become a known standard, analogous to ITSEC and TCSEC. In this paper we don't aim for a standard classification. Hence we focus on relating different segments of the network infrastructure within one (large) organisation to the applications used within the same organisation. Therefore we consider an Information Security Classification which is specific for the organisation. Further research can focus on a standard classification which enables comparison of measures in general.

The Information Security Classification is on the one hand used by application and business process owners to describe their needs. On the other hand this classification has to be used with respect to the network infrastructure. This can be done by relating the Information Security Classification to generic data communication goals that uniquely correspond 
to security measures in the different segments of the infrastructure. Hence the classification should be understandable by network infrastructure owners giving them unambiguous descriptions of what measures to implement to reach a specific classification level.

For clarification purposes we use a simple security classification that consists of three levels of security for the quality aspects Availability, Integrity and Confidentiality. The levels vary from " 1 " (low) to " 3 " (high). This will result in a 3 digit number (AIC) to define the security demands for IT components, including the implemented security measures.

\section{Examples:}

- Security demands for an application: Availability high, integrity medium and confidentiality low results in: $\mathrm{AIC}=321$.

- Implemented security measures in a certain platform: Availability low, integrity medium and confidentiality medium results in: $\mathrm{AIC}=322$.

In a similar manner the necessary security characteristics of the network infrastructure segments can be defined.

The following table is an example of a security classification, which shows the relation between the above mentioned information security classification levels and network infrastructure security measures. The measures are derived from the anticipated goals. Usually the network infrastucture owners can easily translate the examples of measures mentioned in the table to the measures which are necessary for the infrastructure components for which they are responsible. 


\section{Availability}

Level

Anticipated goal

Examples of measures

\begin{tabular}{|c|c|c|}
\hline 3 & $\begin{array}{c}\text { Guaranteed availability }> \\
99.5 \% \text { of service time. } \\
\text { Unavailability of service } \\
\text { related to more than } 50 \% \text { of the } \\
\text { connections will be restored } \\
\text { within } 4 \text { service hours. } \\
\text { Unavailability of less than } \\
50 \% \text { of the connections will be } \\
\text { restored within } 8 \text { service hours. }\end{array}$ & $\begin{array}{c}\text { Duplicate implementation of } \\
\text { central infrastructure. No single } \\
\text { points of failure except for end user } \\
\text { connections. End users connections } \\
\text { belonging to one business process } \\
\text { are patched over two switches. } \\
\text { Service contract with Third Party } \\
\text { Maintenance organisation. }\end{array}$ \\
\hline 2 & $\begin{array}{c}\text { Guaranteed availability }> \\
98 \% \text { of service time. } \\
\text { Unavailability of more than } \\
500 \text { users will be fixed within } 4 \\
\text { service hours. } \\
\text { Unavailability of less than } \\
500 \text { users will be fixed within } 8 \\
\text { service hours. }\end{array}$ & $\begin{array}{c}\text { Partial duplicate implementation } \\
\text { of components. Spare parts for non } \\
\text { duplicate components are available } \\
\text { on-site or contractually agreed } \\
\text { available in time. }\end{array}$ \\
\hline 1 & $\begin{array}{c}\text { Guaranteed availability } \\
98 \% \text { of service time. } \\
\text { Unavailability of more than } \\
500 \text { users is fixed }>4 \text { service } \\
\text { hours. } \\
\text { Unavailability of less than } \\
500 \text { users will be fixed }>8 \\
\text { service hours. }\end{array}$ & \\
\hline
\end{tabular}

Integrity

Level Anticipated goal Examples of measures

\begin{tabular}{|c|c|c|}
\hline 3 & $\begin{array}{c}\text { Change of information flow } \\
\text { is not possible. }\end{array}$ & $\begin{array}{c}\text { Digital signatures or encryption } \\
\text { techniques based on company } \\
\text { accepted algorithms. }\end{array}$ \\
\hline 2 & $\begin{array}{l}\text { Change of information flow } \\
\text { is not possible without physically } \\
\text { breaking into patch panels, } \\
\text { switch boxes etc. }\end{array}$ & $\begin{array}{c}\text { Switching techniques (e.g. } \\
\text { switched Ethernet). }\end{array}$ \\
\hline 1 & $\begin{array}{l}\text { Change of information is } \\
\text { possible. }\end{array}$ & $\begin{array}{c}\text { Shared Ethernet, Internet, } \\
\text { wireless communication. }\end{array}$ \\
\hline
\end{tabular}




\section{Confidentiality}

\begin{tabular}{|l|l|c|}
\multicolumn{2}{c}{ Level } & \multicolumn{1}{c}{ Anticipated goal } \\
\hline 3 & $\begin{array}{l}\text { Eavesdropping information } \\
\text { flow is not possible. }\end{array}$ & $\begin{array}{c}\text { Encryption techniques based on } \\
\text { company accepted algorithms. }\end{array}$ \\
\hline 2 & $\begin{array}{l}\text { Eavesdropping information } \\
\text { flow is not possible without } \\
\text { physically breaking into patch } \\
\text { panels, switch boxes etc. }\end{array}$ & $\begin{array}{c}\text { Switching techniques (e.g. } \\
\text { switched Ethernet). }\end{array}$ \\
\hline 1 & $\begin{array}{l}\text { Eavesdropping information } \\
\text { flow is possible. }\end{array}$ & $\begin{array}{c}\text { Shared Ethernet, Internet, } \\
\text { wireless communication. }\end{array}$ \\
\hline
\end{tabular}

\section{Security classification table}

The benefits of using such a table are:

- Offering infrastructure architects an unambiguous specification of the security level for selecting the necessary set of data communication security measures.

- Offering application owners means of defining the end (user) to end (application) security characteristics of the infrastructure.

- Offering information systems auditors a clearly defined standard for auditing the adequacy of security measures for both applications and infrastructure.

\section{EVALUATING THE CURRENT INFRASTRUCTURE}

The final goal aimed for is to offer a clear view of the security characteristics of all relevant communication paths in a network. To reach this goal, the network segments and their relations will have to be evaluated.

This can be done by the following steps:

- Describe the network and divide the network into different network segments which are linked together;

- Evaluate the security characteristics of each segment and classify the segment with an AIC value;

- Build a matrix containing the security characteristics (AIC values) of the network. From the matrix one can derive the security characteristics of each relevant communication path.

These steps will be explained below. 


\subsection{Drawing the network}

The easiest way to describe the network with the segments and the links in between is to draw up a network diagram. Each segments in the diagram offers a certain security level with respect to the quality aspects availability, integrity and confidentiality, and is within the area of responsibility of one organisational unit. Using the diagram one is able to figure out which possible paths data will travel between the end user and the central application. The measures in the segments of these paths are relevant for the entire path. Hence the security level of the entire path usually will not exceed the security level of each of the segments.

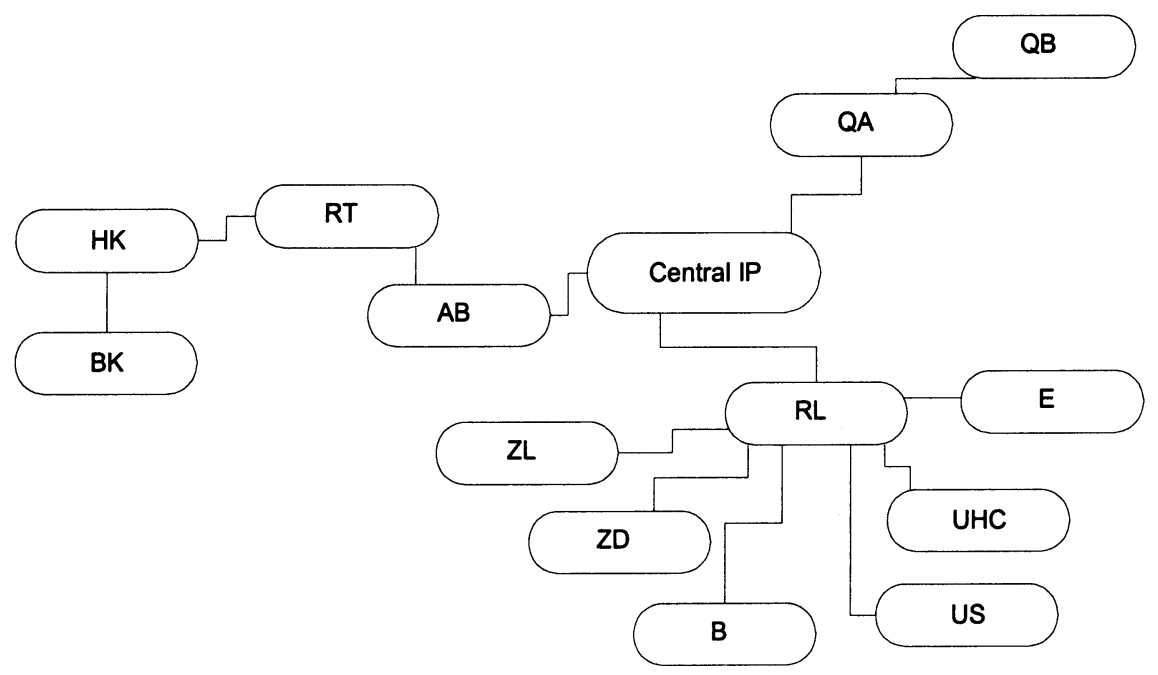

Example network diagram

\subsection{Classifying the segments}

For each network segment the specific security characteristics will have to be evaluated, after which an AIC classification is assigned to the segment. This can be done by matching the implemented measures to the measures mentioned in the security classification table. To identify these measures, the security characteristics of the technical components in the infrastructure will have to be clarified. External information sources such as [TELE97] can be useful to analyse and compare security characteristics in various types of data communication technologies. 
To illustrate the classification of network segments, AIC values are determined for two segments from the diagram (Central IP and HK):

- The Central IP segment does not contain any end users. The infrastructure consists of switched networks (ATM and switched Ethernet) in physically secured areas. The infrastructure does not contain encryption. Since eavesdropping or changing the data flow is not possible without using physical force this results in level 2 values for both integrity and confidentiality.

The Central IP infrastructure is implemented using a duplicate infrastructure which eliminates all single points of failure. Hence the availability measures for level 3 are implemented.

Central IP segment classification: AIC=322.

- The HK segment however does contain single points of failure. Nevertheless the contractual arrangements with an external responsible party guarantee level 2 availability.

The infrastructure contains shared Ethernet which is susceptible to eavesdropping. Integrity and confidentiality only meet level 1.

HK segment classification: $\mathrm{AIC}=211$.

For each segment a similar classification will have to be performed. The correctness of the classification can be tested by an auditor while performing a regular data communication audit.

Note that the classification level for availability usually cannot be increased by using application measures. Hence the resulting value equals the maximum reachable without changing the infrastructure. The classification levels for integrity and confidentiality however can be increased by implementing encryption techniques in the application.

\subsection{Building the matrix}

The ultimate goal of the process is to offer a clear view on the security characteristics of a data communication path between end users and applications systems. This view can be visualised using a matrix. The security classification for all segments is combined into one matrix based on the network diagram. This matrix gives the reader a clear view of the 
security characteristics of a certain data communication path. Hence the matrix can be used to identify easily the security characteristics of the path between end users and the application server.

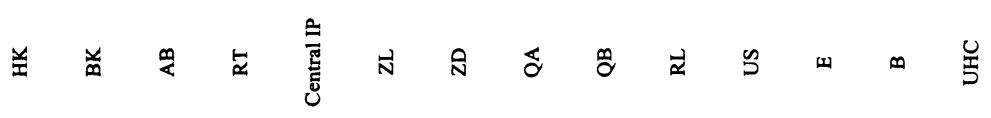

\begin{tabular}{|c|c|c|c|c|c|c|c|c|c|c|c|c|c|c|}
\hline HK & 211 & 211 & 211 & 211 & 211 & 211 & 211 & 211 & 211 & 211 & 211 & 211 & 211 & 211 \\
\hline BK & & 211 & 211 & 211 & 211 & 211 & 211 & 211 & 211 & 211 & 211 & 211 & 211 & 211 \\
\hline$A B$ & & & 322 & 322 & 322 & 322 & 322 & 222 & 211 & 322 & 322 & 322 & 322 & 311 \\
\hline RT & & & & 322 & 322 & 322 & 322 & 222 & 211 & 322 & 322 & 322 & 322 & 311 \\
\hline Central IP & & & & & 322 & 322 & 322 & 222 & 211 & 322 & 322 & 322 & 322 & 311 \\
\hline $\mathrm{ZL}$ & & & & & & 322 & 322 & 222 & 211 & 322 & 322 & 322 & 322 & 311 \\
\hline ZD & & & & & & & 322 & 222 & 211 & 322 & 322 & 322 & 322 & 311 \\
\hline QA & & & & & & & & 222 & 211 & 322 & 322 & 322 & 322 & 311 \\
\hline QB & & & & & & & & & 311 & 311 & 311 & 311 & 311 & 311 \\
\hline RL & & & & & & & & & & 322 & 322 & 322 & 322 & 311 \\
\hline US & & & & & & & & & & & 322 & 322 & 322 & 311 \\
\hline E & & & & & & & & & & & & 322 & 322 & 311 \\
\hline B & & & & & & & & & & & & & 322 & 311 \\
\hline UHC & & & & & & & & & & & & & & 311 \\
\hline
\end{tabular}

Example matrix security characteristics for data communication paths

From this matrix we can read that for an application located on $\mathrm{ZL}$ and the users on HK the maximum reachable AIC value is 211. Furthermore we can see that network availability for an application on ZD with users on QB is limited to level 2 although both ZD and QB have an availability level of 3. This is caused by the intermediate segment QA which only has an availability level of 2 .

Especially in network infrastructures which contain many more segments than the example above, such a matrix offers an unprecedented clarity in the security of the infrastructure. 


\section{USE AND MAINTENANCE}

The matrix of course reflects the security characteristics at a certain point in time. Hence the maintenance of the matrix is essential for the usefulness. The network infrastructure managers should treat it as a quality aspect and communicate it to the relevant parties. Furthermore, the managers can use the information to plan future enhancements in the security measures of the network infrastructure.

Information system owners can use the approach to assess the total security level of an application including its infrastructure. Therefore security levels will have to be communicated to information system owners. This should be done by incorporating the security classification levels as part of the quality levels in the service level management process. Hence it should appear in the standard service catalogue and service level agreements. The information system owners should use the information from the service catalogue as one of the sources to evaluate the need for additional measures. Hence it may result in additional application measures or requests for changes with respect to the infrastructure. The results of these requests should, after negotiation, become part of the service level agreement.

The approach is also useful for auditors [MURP90]. When auditing applications, information systems auditors can use the approach to evaluate the security characteristics of the communications paths between end users and application servers. This information is the starting point for defining the necessary compensating application measures which need to be implemented. When auditing network infrastructures, data communication auditors can use the approach to define a suitable set of security measures for components which are part of the audited network infrastructure. The matrix can be used as a standard for audits of the network infrastructure components. On the other hand a network infrastructure audit has to evaluate the process leading to the security matrix. This should be done to check whether all relevant parties implement their piece of the puzzle correctly.

\section{CONCLUSION}

The data communication infrastructure has a crucial impact on the security of today's IT dependent business processes. The direct translation of the business processes needs into requirements imposed on the infrastructure is virtually impossible due to the number of applications using the 
infrastructure and the complexity of the infrastructure. However, with a structured approach as described in this paper it is possible to visualise the implemented security characteristics and to clarify the necessity for additional security measures. The approach can support cost effective management of the security of data communication infrastructures.

\section{REFERENCES}

[CCTA] CCTA Risk Analysis and Management Methodology (CRAMM), Central

Computer and Telecommunications Agency, UK

[COP] Code of Practice for Information Security Management, BS7799, 1995

[ECC91] European Communities Commision (1991), Information Technology Security Evaluation Criteria (ITSEC).

[NCSC85] NCSC (1985), Department of Defence trusted computer system evaluation (TCSEC), Orange book.

[TELE97] Telematica (1997), Samson bedrijfsinformatica.

[MURP90] Murphy, Michael A., Parker, Xenial Ley (1990), Handbook of EDP auditing, Warren, Gorham \& Lamont. 